\title{
Health is Sexy for Girls
}

\author{
Katie MacEntee
}

$\cos 80$

In the call for articles for this special issue on girls' health, we highlighted that '[g]irls' health is an ongoing and evolving issue with ties that go beyond medical analyses to include a wide array of social, educational, political, and environmental discourses (among others!).” That a number of different perspectives might contribute to or strengthen the interdisciplinary focus of an issue as crucial as girls' health was important to me as guest editor. This issue demonstrates that the relationship of girlhood to health-sexual health, in particular-is of critical concern to us all. It is an area full of challenges and barriers, most of them, as is evident in this issue, understood and often expressed by girls themselves. The articles presented here point to the many perspectives from which to approach this topic. Girls' sexual health is linked to an array of intersecting issues including the pedagogical influences of popular romance literature; the ways in which girls use blogs to construct counter narratives about their sexual identity; how girls' increased inclusion in citizenship discourses can increase their capacity to address sexual objectification; what girls do to negotiate power within their heterosexual relationships; how barriers to water access in Africa can lead to the awareness of the risks - which range from being perceived to be promiscuous to being raped - that young women face; as well as how the (mis)management of menstruation can affect girls' education. This issue points to the global and local specifics of sexual health, and to health more generally. The concerns discussed here are geographically wideranging: Cameroon, Lesotho, Australia, the United States, and Canada provide the settings - some urban and others rural. The authors present a wide range of methodologies from which they explore girls' health: literary analysis; autoethnography; and participatory methods such as digital storytelling, mediamaking, listening to what young people have to say in various research paradigms, blogging, and photovoice.

The issue begins with Elizabeth Bullen's article, "Erotic Capital, Popular Pedagogy and Healthy Adolescent Female Sexuality," —a liter- 
ary analysis of two chick lit texts in the genre of young adult romance. As she points out, this genre, part of a multi-million dollar industry, is read primarily by girls and young women. Bullen unpacks the contradictory nature of the messages expressed in this kind of novel-messages of liberation and subjugation of female sexuality are offered side by side. She warns that the ever-growing popularity of chick lit may be promoting in girls a misplaced supposedly postfeminist ideal of sexuality and desire and she points to the need to "take into account the tensions between feminist concepts and popular culture constructions of girl desire and, likewise, the tensions between popular pedagogies and a feminist sex education, including its ideals for a healthy adolescent female sexuality."

Aline Gubrium and Gloria DiFulvio's article, "Girls in the World: Digital Storytelling as a Feminist Public Health Approach" sees girls themselves as central to addressing issues of sexual health and safety. Their research methodology uses digital storytelling as a tool for addressing issues of girls' health in community settings. Drawing on their work in Anthropology and Public Health, Gubrium and DiFulvio focus on knowledge production and the ways in which a feminist approach to this can offer a humanistic perspective on girls' health. Highlighting their work with Latina girls in the eastern USA, they include a detailed analysis of two of the digital stories from a pilot project called A Girl in the World. The discussion related to the digital storytelling workshops and the analysis of the stories themselves offer a rich contribution to the literature on participatory methodologies in relation to girls' health.

Like Gubrium and DiFulvio, Lyn Mikel Brown, in her article, "We're Taking Back Sexy: Girl Bloggers SPARK a Movement and Create Enabling Conditions for Healthy Sexuality," emphasizes the importance of having girls front and center in research that relates to them. Reporting on her work with young women involved in the SPARK movement- "an intergenerational movement that raises awareness about, and pushes back against, the sexualization of women and girls in the media"-Brown looks at the potential for blogs to provide a space in which girls can counter media representations of girls' sexuality. This work also models how peer support and peer education amongst girls can help in the construction and reclamation by young women of what it means to be sexy. Drawing on the SPARK girls' blogs, Brown highlights the ways in which young women understand, contest, and 
construct their everyday lives as it relates to their own sense of what constitutes being sexy.

Then with Caroline Caron's article, "Getting Girls and Teens into the Vocabularies of Citizenship," we move towards a consideration of girls' sexuality and the notion of what Caron terms 'political health' and its relationship to citizenship, given the apparent invisibility of adolescent girls particularly as far as feminist theory and citizenship studies are concerned. Building on her own participatory work with Francophone girls and young women in Quebec where the issues of hypersexualization have been taken up in the mainstream media, Caron offers a compelling argument advocating not only mediamaking work with girls but also the need to recognize the value of girls' participation in studying their own lives. Caron points to the need for "girlhood studies to politicize its vocabulary so that teenage girls can become part of us rather than women-to-become in feminist citizenship studies and others areas of inquiry in which youth citizenship is being re-theorized." For her, "such a politicization broadens what girls' health entails to include their political healthiness."

Marion Doull and Christabelle Sethna in "Subject, Object, or Both? Defining the Boundaries of 'Girl Power"' take up the current debate within third wave feminism about whether girl power is an oppressive or an empowering notion for girls and young women. They focus on the interrelated issues of power and sex by arguing that girls' experiences of sexual relationships are too often overlooked and excluded from academic debate. Talking with girls, and using, most significantly, an asset-based approach, about their personal understandings of power and sex in heterosexual relationships allows the authors to discuss the ways in which girls can choose to walk a strategically considered line between objectification and subjectification in these sexual relationships and how they negotiate the complex power dynamics between themselves and their partners. As Doull and Sethna so cogently point out, "We need to question the choices women are offered to be powerful in their heterosexual relationships and beyond rather than critiquing the choices they make."

"Fetching Water in the Unholy Hours of the Night: The Impacts of a Water Crisis on Girls' Sexual Health in Semi-urban Cameroon," by Jennifer Thompson, Fidelis Folifac and Susan Gaskin explores how, in a school competition on water access and the environment in Cameroon, 
one of the main topics of discussion and concern was that of girls and their sexual behaviors. That participating youth perceive young girls living in the midst of a water shortage-often out for hours looking for working water taps or for alternative water sources-as so sexually promiscuous as to take advantage of the conditions of this quest for water was an unexpected research finding. As the authors explain, water access challenges, typically, are addressed through a development perspective which is concerned with infrastructure, such as the need for more water pumps. Gender is most often left out of this equation, and so is health, outside of its relationship to water quality and sanitation. A consequence of the competition was to make public the water distribution schedule outlining the timing and availability of working water pumps. This relatively simple policy change means that the community can plan for water shortages and girls do not face as much risk during their water collection activities.

Using an autoethnographic method in "Growing Up a Girl in a Developing Country: Challenges for the Female Body in Education" Mathabo Khau explores the connections between menstruation and schooling in Lesotho. She offers a blueprint of how menstruation can be managed and negotiated in a school setting. The experiences recounted in this article are at times all too familiar to many women the world over while at other times the personal reflections point to contextually specific experiences and barriers to girls' education in an impoverished African context. Certainly, if issues related to menstruation are ignored this can have a major impact on girls' ability to attend, participate, and excel in some schooling environments. And as Khau's argument goes on to stress, teachers need to be made explicitly aware and must be trained to attend to these sorts of sex-specific experiences in a way that is culturally appropriate. As Khau clearly states, what is required is a "concerted effort from all stakeholders in education to ensure that schools are female friendly." Providing free sanitary towels for students and improving toilet facilities are starting points from which improve the experiences of girls in school. Recognizing in school curricula and policy what is essential to girls' health will further promote gender equality in schools.

In her visual essay, "Let's Talk About Sex: Photo Gallery" Ciann Wilson discusses the use of photovoice to engage girls in the exploration and expression of their individual understandings of sexual iden- 
tity and health. Having worked with a group of girls living in the lower income neighbourhood of Jane and Finch, Toronto, Wilson draws our attention to girls who are marginalised on the bases of sex, age, and race. The photos presented here, accompanied by narratives written by the photographer-participants, illustrate the girls' understanding of how to manage their own sexual health. These girls, as we can so clearly see, are active participants in the construction of their sexual identity. They negotiate their sexual health issues with determination, courage and remarkable self-awareness. That these photos and narratives are, and will be, made available for viewing by a wide audience is of the utmost importance: these insights beg to be shared.

Two book reviews extend the theme of girls and health. Shelley Peterson reviews J.L.Power's young adult novel This Thing Called the Future. The story, set in a township near Pietermaritzburg in South Africa, addresses the issue of HIV\&AIDS and the ways in which 'this thing called the future' is also about gender and the high risks of infection for girls and women in cases of rape and sexual infidelity. The young protagonist, fourteen-year-old Khosi is caught between the old world of her grandmother who believes in witchcraft and the new world of her mother who believes in science and medicine. For Khosi the issues are far more complex than an either/or choice and the reviewer points to these nuances in useful extracts from the text of the novel.

Finally, Nicole Power's review of Nicole Landry's book The Mean Girl Motive: Negotiating Power and Femininity, considers how Landry sees the phenomenon of the mean girl in popular culture being lived out in the lives of real girls. Landry argues that western girls have limited access to power within their social milieus: being popular is seen to be the most coveted social position. Once in a position of social power, girls must fight to maintain this position by acting out the 'mean girl' role. When it comes to girls' mental health and well-being, as the review makes clear, we need more work like Landry's which "critiques overly simplistic and psychologizing explanations of girl aggression."

As noted above, that so many of the authors chose to take up issues related specifically to sexual health suggests that this is an area of enormous current concern. One the one hand, the multiplicity of contexts from which the topic has been addressed in this issue reveals the significance of investigating these topics at the individual or local level. On the other hand, the overarching similarities of insight amongst the 
pieces also imply the existence of systemic factors, which, at a global level, deserve more investigation. The use of participatory methods to ensure the inclusion of the active voices of girls in knowledge production is a noteworthy contribution. Although these methods are becoming increasingly popular in many fields of inquiry, more work on which methodologies are best used to do this, how they interact, contest, or as Caroline Caron asks, contradict one another, are questions for further exploration. There is potential for these methods to be scaled up even as they are becoming recognised for their ability to provide evidence that can ultimately inform realistic, cost effective, and sustainable policy change. As Gubrium and DiFulvio contend in relation to digital storytelling the "process could be used in public health and related health policy-making endeavors, thus shifting from a personally emancipating process to a political public health process, in which stories shared within groups and communities work to deepen dialogue for action." We can extrapolate from this: much of the work reported on in this issue would provide compelling evidence-based imperatives for the design, creation and implementation of various girl-friendly and girlhealthy policies. Participatory methodologies offer rich contributions to academic knowledge construction as well as to on-the-ground action. Khau's autoethnographic work contributes to the current conversation about creating an appropriate policy to address girls' menstrual hygiene challenges in lower income settings. Thompson and her colleagues, in using a participatory methodology demonstrate effectively how the inclusion of young peoples' stories can lead to a relatively simple policy development that has had a real impact on girls' lives. The overall contribution of all this is a deepening of our understanding of many aspects of the topic of health as it relates to girls and I want to thank Girlhood Studies for giving me the opportunity to be a part of this exciting new awareness of what remains, still, to be done. My thanks, too, to Claudia Mitchell and Ann Smith for their invaluable help. 